\title{
Salivary Duct Carcinoma of the Submandibular Gland: A Case Report and Review of the Literature
}

\author{
Ilson Sepulveda ${ }^{\mathrm{a}, \mathrm{b}, \mathrm{g}}$, Francisco Rivas-Rodriguez ${ }^{\mathrm{c}}$, Pamela Villalobos ${ }^{\mathrm{d}}$, \\ Carola Monsalvez ${ }^{\mathrm{d}}$, Alvaro Compan ${ }^{\mathrm{e}, \mathrm{f}}$, J. Patricio Ulloa ${ }^{\mathrm{e}}$ f
}

\begin{abstract}
We report a patient who presented with swelling to the left submandibular region. Imaging studies revealed an expansive heterogeneous process. The patient underwent tumor resection and a biopsy confirmed the presence of a salivary duct carcinoma. Additional treatment included chemotherapy and the patient is currently receiving palliative and supportive care for advanced metastatic disease.
\end{abstract}

Keywords: Salivary duct carcinoma; Mammary; Computed tomography; Magnetic resonance; Recurrence; Metastasis; Surgical; Radiation therapy; Chemotherapy

\section{Introduction}

Salivary duct carcinomas (SDCs) are rare and aggressive salivary gland tumors that predominantly occur between the fifth and sixth decades of life, predominantly in males. These head and neck tumors are locally aggressive, with early recurrence and distant metastases. Imaging studies are essential for preoperative staging and surgical planning. The treatment of choice is complete surgical resection and adjuvant radiation therapy.

\section{Case Report}

A 51-year-old man with a medical history of arterial hyperten-

Manuscript submitted November 27, 2019, accepted December 9, 2019

${ }^{a}$ Finis Terrae University School of Dentistry, Santiago, Chile

${ }^{b}$ Radiology Department, Otorhinolaryngology-Head \& Neck Surgery, Maxillofacial Services, General Hospital of Concepcion, Concepcion, Chile

'Radiology Department, Division of Neuroradiology, University of Michigan Health System, Ann Arbor, MI, USA

dPathology Department, General Hospital of Concepcion, Concepcion, Chile 'Otorhinolaryngology-Head \& Neck Surgery, General Hospital of Concepcion, Concepcion, Chile

fUniversity of Concepcion School of Medicine, Concepcion, Chile

${ }^{g}$ Corresponding Author: Ilson Sepulveda, ENT-Head and Neck Surgery Service, General Hospital of Concepcion, San Martin Av. 1436, Concepcion, Chile. Email: isepulvedaa@uft.edu

doi: https://doi.org/10.14740/jmc3396 sion, obesity and smoking presented to the Ear, Nose and Throat clinic (ENT) complaining of progressive left submandibular swelling of 10 years duration. No pain or neurologic deficits were present. A sonography was performed that showed a solid hypoechogenic mass of $3.7 \mathrm{~cm}$ diameter, hyperechogenic calcium deposits within, and supracentimetric lymphadenopathies in II and V ipsilateral ganglionic levels (Fig. 1).

A neck-chest-abdomen computed tomography (CT) scan was performed, revealing an expansive-infiltrative, heterodense solid mass on the left submandibular gland, that measured $3.8 \mathrm{~cm}$ in diameter. The mass displayed irregular contours with central hypodense areas following intravenous contrast administration, suggestive of necrosis and calcifications within. The mass was invading the ipsilateral medial pterygoid and platysma muscles. Lymphadenopathy was observed with hypodense center in the left II-V ganglionic level as large as 11 $\mathrm{mm}$. In addition, CT revealed the presence of nodules in the liver suggestive of metastatic disease (Fig. 2).

A biopsy was performed under general anesthesia revealing an SDC. The Head and Neck Tumor Board reviewed the case, and recommended surgical excision and neck dissection. The postsurgical biopsy confirmed the presence of an SDC with infiltration of adjacent structures and positive lymphovascular/perineural invasion. Immunohistochemistry tests were positive for androgen receptor (AR+), Cerb-B2, GATA 3 and CK7 and negative for P63, KHMW, CK5/6 and P40 (Fig. 3).

Follow-up by The Head and Neck Tumor Board where it was staged as a T3N2M1 resulted in the recommendation of palliative chemotherapy. He received six cycles of carboplatin and taxol, showing good tolerance and clinical local improvement. At the end of the treatment, neck-chest-abdomen-pelvis CT examinations were performed, revealing the presence of progressive liver nodules, and thoracic, lumbar, and sacral spine bone lytic lesions consistent of extensive metastatic disease (Fig. 4). The patient was referred to the pain control and palliative care program.

\section{Discussion}

Salivary gland cancers are rare, accounting for $<1 \%$ of all malignancies $[1,2]$ and less than $5 \%$ of all head and neck malignancies. SDC accounts for approximately $0.2-2 \%$ of all salivary gland neoplasms. The first case of SDC was described by Kleinsasser et al in 1968 who described the distinct histologic similarity to ductal carcinoma of the breast, and was rec- 


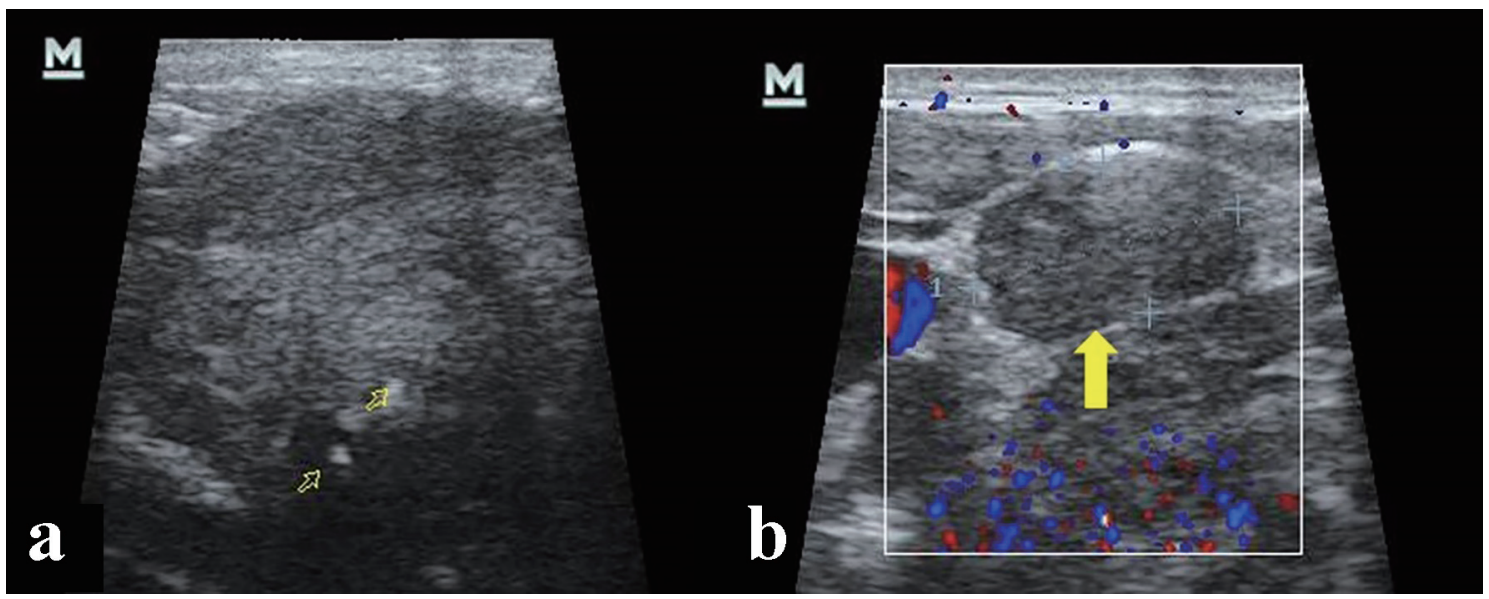

Figure 1. Cervical ultrasonography. (a) Hypoecogenic, heterogeneous solid mass and calcium deposit within (open yellow arrows). (b) Lymphadenopathy in $\mathrm{V}$ ganglionic level (yellow arrow).

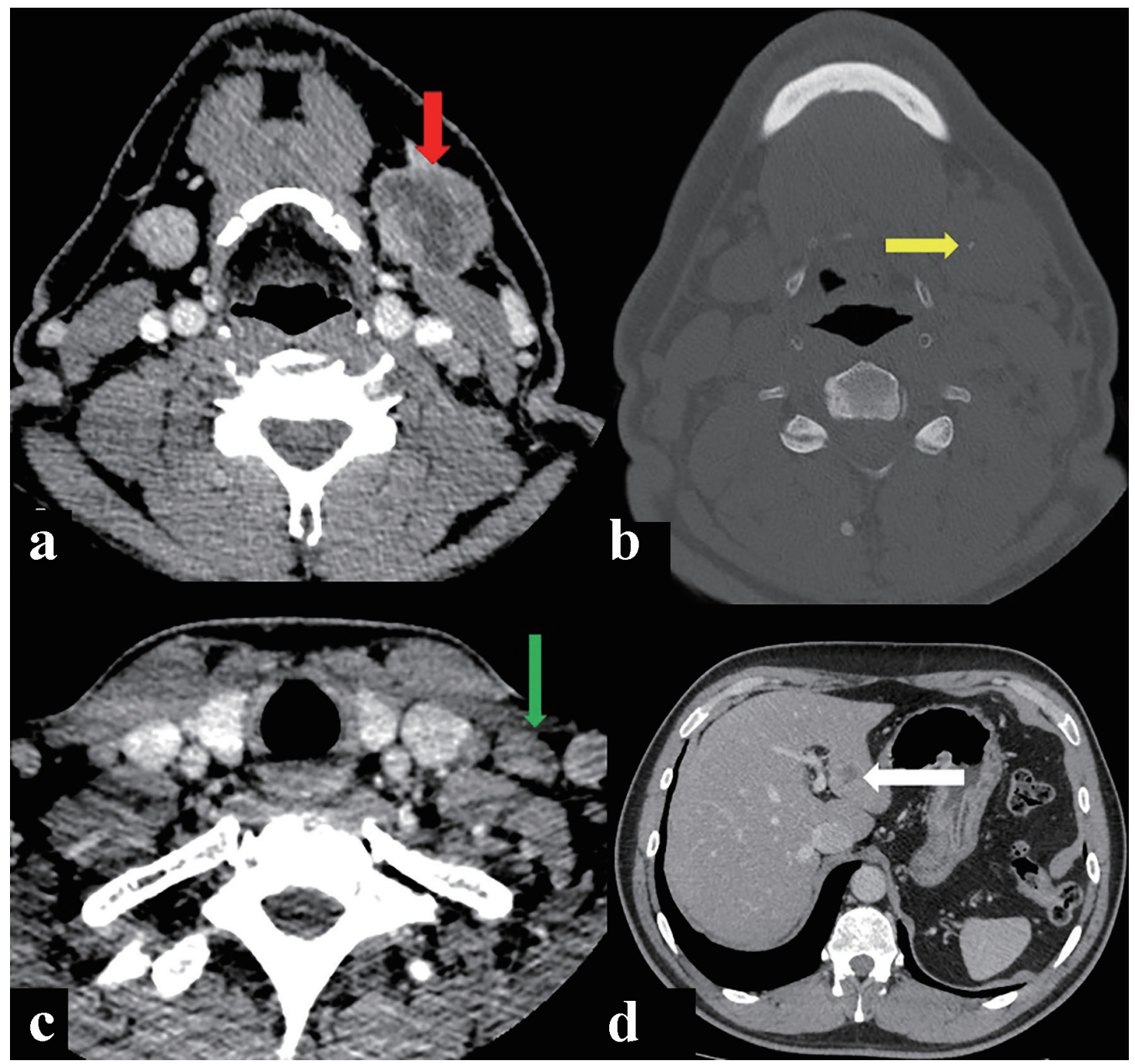

Figure 2. Computed tomography (CT) showing a primary left submandibulary mass (red arrow) with little calcifications within (yellow arrow), lymphadenopathy by size and morphology criteria in $\mathrm{V}$ ganglionic level (green arrow), and metastatic liver nodule (with arrow). (a, c, d) CT soft tissues window after intravenous contrast injection. (b) CT bone window axial view. 


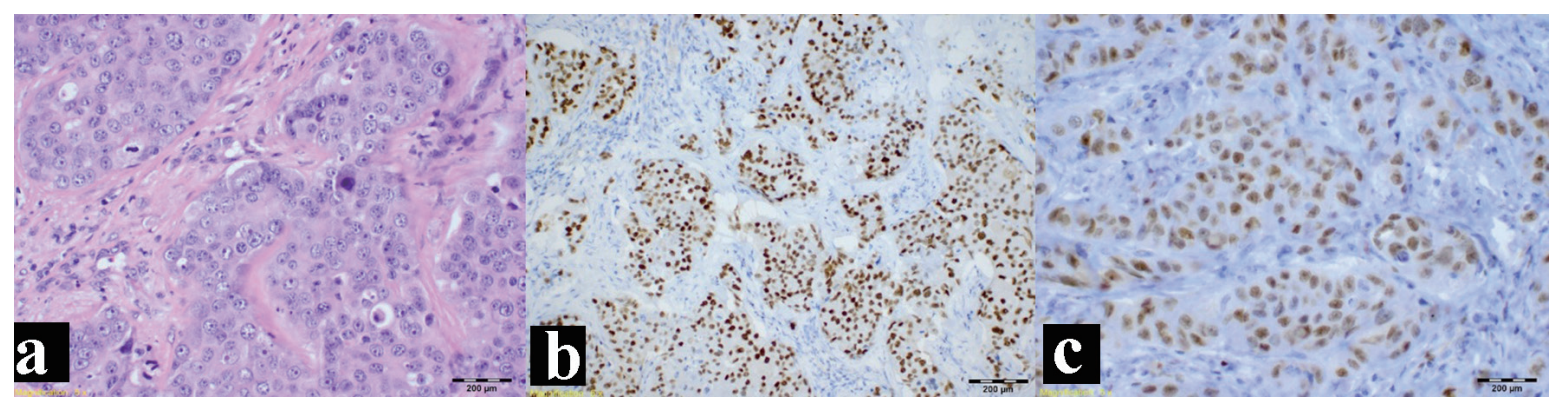

Figure 3. Immunohistochemistry study. (a) Hematoxylin and eosin stain. (b) Positive androgen receptor stain. (c) Positive GATA 3 stain.

ognized as a distinct entity by the World Health Organization (WHO) in 1991 [3-5].

According to the 2017 WHO classification, SDC is an aggressive epithelial malignancy resembling high-grade mammary ductal carcinoma. It can occur de novo or as a carcinoma expleomorphic adenoma (CAxPA), the latter being a recent finding $[6,7]$. "Low-grade SDC" and "SDC in-situ" of the former WHO classification are now named low-grade and highgrade intraductal carcinoma (IDC), respectively [8].

The male to female ratio reported is approximately $3: 1$; in the fifth or sixth decades of life, with the average age of occurrence at 60 years, SDC is more common [9-11], more common in the parotid gland (78-83\%), whereas submandibular location (12\%) and minor salivary glands (10\%) are rarely affected. SDCs are made up of ductal and cribriform structures lined with epithelial cells with marked cytologic atypia. A Roman bridge like appearance and comedo-necrosis are typically observed in SDC, which strongly resembles ductal carcinoma of the breast [12]. The grading scheme that characterizes SDCs into four histologic patterns is as follows: sarcomatoid, mucinrich, micropapillary and basal-like [5]. The histopathologic diagnosis of SDC was based on hematoxylin and eosin stains and confirmed by immunohistochemical staining which have shown that SDC typically demonstrates epithelial markers, including cytokeratin, epithelial membrane antigen (EMA) and carcinoembryonic antigen (CEA) $[9,12]$.

Clinically, SDC is now well recognized as an aggressive malignancy, frequently presenting as stage IV disease [11], present as a rapidly enlarging firm mass with a poor prognosis that is characterized by early lymph node invasion, distant me- tastases (brain, liver, adrenals, bone, skin and thyroid) (50\%), high rate of local recurrence $(50 \%)$ and ultimately a low survival rate, 3 years median $[9,13,14]$. Perineural and lymphatic invasion, further indicators of a poor prognosis, are frequently present in approximately $57 \%$ to $69 \%$ and $61 \%$ to $70 \%$ of patients, respectively [12]. Based on the study of SDC of the major salivary gland, tumor size $>3 \mathrm{~cm}$ and age $>50$ years old have been proposed to be related to poor prognosis [15].

SDC is usually described as an ill-defined, infiltrative mass with frequent necrosis on $\mathrm{CT}$ and magnetic resonance (MR) images [16]. The presence of calcification in an ill-defined salivary gland mass may be a good CT criterion that raises the possibility of SDC [17]. MR imaging has widely been used for differentiation of benign and malignant tumors of the salivary gland. The lower the signal intensity on T2-weighted images, the higher the possibility of high-grade malignancy [18]. According to Wang et al [19], an apparent diffusion coefficient (ADC) value $<1.22 \times 10^{-3} \mathrm{~mm}^{2} / \mathrm{s}$ was one of the criteria for predicting malignancy. Yabuuchi et al [20] reported that a short time of peak enhancement, $<120 \mathrm{~s}$, and a low washout ratio, $<$ $30 \%$ (type B), on dynamic MR images were useful criteria for the diagnosis of malignant tumor. The focus in SDC that shows gradual upward enhancement on dynamic MR images, low $\mathrm{ADC}$ value and hypointensity on short-tau inversion recovery (STIR) and T2-weighted images may be a clue for making a diagnosis of SDC and separating SDC from the more common malignant salivary gland tumors such as mucoepidermoid carcinoma and adenoid cystic carcinoma [21].

The treatment of choice is surgery according to the tumor site and its extent. Postoperative radiotherapy of $60-66 \mathrm{~Gy}$

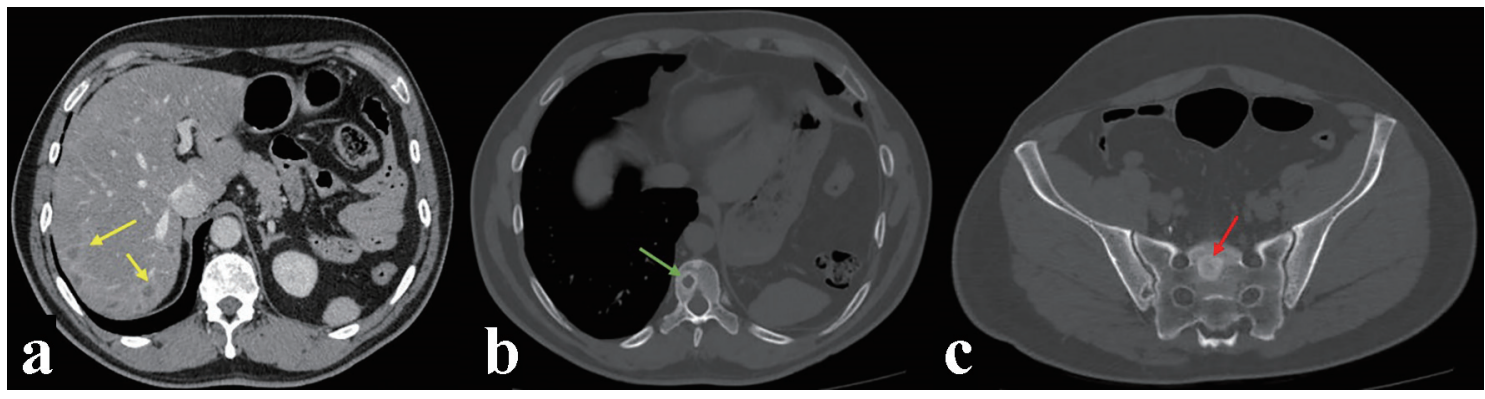

Figure 4. Computed tomography (CT) control imaging after treatment showing liver nodules (yellow arrows), and thoracic (green arrow) and sacral (red arrow) spine bone lytic lesions consistent of metastatic disease. (a) CT soft tissues window after intravenous contrast injection. (b, c) CT bone window axial view. 
was given when any of the resection margins was positive or equivocal and/or lymph node metastasis was pathologically positive [3]. Chemotherapy is used for palliation in metastatic form of the tumor [8]. Molecularly targeted therapy against HER2 protein with anti-HER2 monoclonal antibodies has been suggested to be contributive as a therapeutic target for patients with HER2/neu-positive SDC $[11,13]$.

Tumor stage is an important indicator of overall survival and early cancer recurrence. The overall mean survival of these patients was reported to be approximately 36 to 56 months. The 5-year disease-free survival rates for stage I, II, III and IV SDCs were approximately $42 \%, 40 \%, 30 \%$ and $23 \%$, respectively [13]. Local recurrence occurred in $48 \%$ of patients at 17.4 months after initial treatment. Distal metastases arose in $48 \%$ after 29 months [22].

\section{Conclusion}

$\mathrm{SDC}$ is a rare and distinctive neoplasm with an aggressive behavior such as regional nodal and early distant metastasis, local recurrence and significant mortality. Although CT and MR imaging findings are nonspecific, the presence of calcifications on CT scans, low ADC value and marked low signal intensity on T2-weighted MR images may be radiologic features to suggest SDC. The treatment of choice is complete surgical excision and neck dissection; adjuvant radiation therapy is reserved for the extensive local infiltrative and lymph node invasion related to this malignancy (advanced form). HER2targeted therapies may therefore be a novel and effective future treatment choice for certain SDC patients. Systemic chemotherapy is reserved for unresectable locoregional recurrence or where there are distant metastases for palliative management.

\section{Acknowledgments}

None to declare.

\section{Financial Disclosure}

None to declare.

\section{Conflict of Interest}

The authors have no conflict of interest to disclose.

\section{Informed Consent}

Not applicable.

\section{Author Contributions}

RS and FRR contributed to revision of bibliography and imag- ing concepts, and revision and edition of the manuscript. PV and $\mathrm{CM}$ contributed to histological antecedents, photography and biopsy. AC and JPU contributed to clinical and surgical information.

\section{References}

1. Chandrasekar C, Salati N, Rao L, Radhakrishnan R. Salivary duct carcinoma in the mandibular anterior region: The role of immunohistochemical markers in its definitive diagnosis. J Oral Maxillofac Pathol. 2016;20(3):505509.

2. Iqbal MS, Shaikh G, Chatterjee S, Cocks H, Kovarik J. Maintenance therapy with trastuzumab in her2 positive metastatic parotid ductal adenocarcinoma. Case Rep Oncol Med. 2014;2014:162534.

3. Otsuka K, Imanishi Y, Tada Y, Kawakita D, Kano S, Tsukahara K, Shimizu A, et al. Clinical Outcomes and Prognostic Factors for Salivary Duct Carcinoma: A MultiInstitutional Analysis of 141 Patients. Ann Surg Oncol. 2016;23(6):2038-2045.

4. Kim TH, Kim MS, Choi SH, Suh YG, Koh YW, Kim SH, Choi EC, et al. Postoperative radiotherapy in salivary ductal carcinoma: a single institution experience. Radiat Oncol J. 2014;32(3):125-131.

5. Udager AM, Chiosea SI. Salivary Duct Carcinoma: An Update on Morphologic Mimics and Diagnostic Use of Androgen Receptor Immunohistochemistry. Head Neck Pathol. 2017;11(3):288-294.

6. Gilbert MR, Sharma A, Schmitt NC, et al. A 20-year review of 75 cases of salivary duct carcinoma. JAMA Otolaryngol Neck Surg. 2016;142:489-495.

7. El-Naggar AK, Chan J, Takata T, et al. Pathology and genetics of head and neck tumours. In: WHO classification of tumours. Lyon: IARC Press; 2017.

8. D'Heygere E, Meulemans J, Vander Poorten V. Salivary duct carcinoma. Curr Opin Otolaryngol Head Neck Surg. 2018;26(2):142-151.

9. Acharya S, Padmini S, Koneru A, Krishnapillai R. Intraoral salivary duct carcinoma: A case report and a brief review. J Oral Maxillofac Pathol. 2014;18(Suppl 1):S121-127.

10. Xie S, Yang H, Bredell $M$, Shen S, Yang H, Jin L, Zhang S. Salivary duct carcinoma of the parotid gland: A case report and review of the literature. Oncol Lett. 2015;9(1):371-374.

11. Limaye SA, Posner MR, Krane JF, Fonfria M, Lorch JH, Dillon DA, Shreenivas AV, et al. Trastuzumab for the treatment of salivary duct carcinoma. Oncologist. 2013;18(3):294-300.

12. Hsu CC, Li WY, Chu PY. Salivary duct carcinoma of the supraglottis with a distinct presentation: A case report and literature review. Medicine (Baltimore). 2018;97(11):e0095.

13. Imaue $\mathrm{S}$, Tomihara $\mathrm{K}$, Hamashima $\mathrm{T}$, Tomizawa $\mathrm{G}$, Nomura K, Sasahara M, Noguchi M. Successful multimodal treatment of intraoral salivary duct carcinoma in a patient with multiple lymph node metastases: a case re- 
port. World J Surg Oncol. 2017;15(1):18.

14. Campos-Gomez S, Flores-Arredondo JH, Dorantes-Heredia R, Chapa-Ibarguengoitia M, de la Pena-Lopez R. Case report: anti-hormonal therapy in the treatment of ductal carcinoma of the parotid gland. BMC Cancer. 2014;14:701.

15. Jayaprakash V, Merzianu M, Warren GW, Arshad H, Hicks WL, Jr., Rigual NR, Sullivan MA, et al. Survival rates and prognostic factors for infiltrating salivary duct carcinoma: Analysis of 228 cases from the Surveillance, Epidemiology, and End Results database. Head Neck. 2014;36(5):694-701.

16. Okada F, Honda K, Ando Y, Nakayama T, Sai M, Tanoue S, Matsumoto S, et al. Salivary duct carcinoma of the extra-glandular segment of Stensen's duct: radiological findings and pathological correlation (2008: 10b). Eur Radiol. 2009;19(1):254-257.

17. Weon YC, Park SW, Kim HJ, Jeong HS, Ko YH, Park IS, Kim ST, et al. Salivary duct carcinomas: clinical and CT and MR imaging features in 20 patients. Neuroradiology.
2012;54(6):631-640.

18. Som PM, Biller HF. High-grade malignancies of the parotid gland: identification with MR imaging. Radiology. 1989;173(3):823-826.

19. Wang J, Takashima S, Takayama F, Kawakami S, Saito A, Matsushita T, Momose M, et al. Head and neck lesions: characterization with diffusion-weighted echo-planar MR imaging. Radiology. 2001;220(3):621-630.

20. Yabuuchi H, Fukuya T, Tajima T, Hachitanda Y, Tomita K, Koga M. Salivary gland tumors: diagnostic value of gadolinium-enhanced dynamic MR imaging with histopathologic correlation. Radiology. 2003;226(2):345-354.

21. Motoori K, Iida Y, Nagai Y, Yamamoto S, Ueda T, Funatsu $\mathrm{H}$, Ito $\mathrm{H}$, et al. MR imaging of salivary duct carcinoma. AJNR Am J Neuroradiol. 2005;26(5):1201-1206.

22. Jaehne M, Roeser K, Jaekel T, Schepers JD, Albert N, Loning T. Clinical and immunohistologic typing of salivary duct carcinoma: a report of 50 cases. Cancer. 2005;103(12):2526-2533. 